\title{
Experimental induction of blunt chest trauma in mice: A modified approach with evaluation in dummies and cadavers
}

\author{
DENIS HÖFER ${ }^{1}$, CHRISTINA KÖRBEL ${ }^{2}$, MATTHIAS W. LASCHKE ${ }^{2}$, NILS T. VEITH $^{3}$, \\ TIM POHLEMANN $^{3}$, REINHARD KAPPL ${ }^{4}$ and THOMAS TSCHERNIG ${ }^{1}$
}

\author{
Departments of ${ }^{1}$ Anatomy and Cell Biology, ${ }^{2}$ Clinical and Experimental Surgery, ${ }^{3}$ Trauma, Hand and \\ Reconstructive Surgery and ${ }^{4}$ Biophysics, Saarland University, D-66421 Homburg/Saar, Germany
}

Received December 12, 2019; Accepted July 1, 2020

DOI: $10.3892 / \mathrm{etm} .2020 .9156$

\begin{abstract}
Experimental chest trauma or blunt thoracic trauma using a blast wave mechanism is well established in animal models. The aim of the present study was to establish a complementary, murine experimental chest trauma model precisely defined by physical data and calculations. For this purpose, a device was developed using a dropped weight and physical properties, including velocity, energy and impact, were calculated. The device allowed for the maximum depth of impression to be measured. The device was first tested using blocks of modelling clay and was then applied to mouse cadavers. X-ray and dissection were performed to check for bone fractures and organ injuries following blunt chest traumas of increasing impact. Lesions and hemorrhages were observed in mouse cadavers which sustained a force equivalent to the energy of $\sim 1 \mathrm{~J}$.
\end{abstract}

\section{Introduction}

Blunt chest trauma leads to death in up to $60 \%$ of patients after car accidents (1). Small and large animal models of blunt chest trauma are necessary to develop novel therapeutic approaches. Previously, a blast wave generator was developed to consistently induce chest traumata in rats (2). In the last decade, mice have primarily been used as experimental animals for studies investigating blunt chest trauma in order to explore the abundant immunological and genetic tools in this species. A previous study reported the physiological and immunological characterization of a long-term model of blunt chest trauma in mice using a blast wave generator (3). Another approach for inducing blunt chest trauma is dropping a weight from a defined height, which has been used in multiple rat and murine trauma models (4-6).

Correspondence to: Professor Thomas Tschernig, Department of Anatomy and Cell Biology, Saarland University, 100 Kirrberger Street, D-66421 Homburg/Saar, Germany

E-mail: thomas.tschernig@uks.eu

Key words: blunt chest trauma, mouse model, depth of impression
In the present study, a device was used to simulate the frontal impact of a car on the human body. A block of metal of varying weight dropped from different heights was used to induce defined trauma, ranging from contusions up to severe lesions. In contrast to non-elastic material, real bodies display post mortem recoil after impact. The present device was developed to measure the maximum depth of impression. To illustrate the effect of the maximum depth of impression on blunt trauma against thoracic organs, including the heart, lungs and big vessels, a schematic drawing is presented in Fig. 1. The device was tested on plasticine dummies as well as on mouse cadavers. Cadavers were used to obtain preliminary results to minimize the use of living animals. The present study aimed to demonstrate that the new device developed was able to generate reproducible impact trauma, which may be calculated and physically defined. This device may aid in subsequent impact studies and improve the current knowledge on the effect of maximum depth of impression on blunt chest trauma.

\section{Materials and methods}

Blunt chest trauma device. The device used in the present study was a block of metal of defined weight that was dropped from a defined height on the dummies or mouse cadavers. A schematic of the construction is presented in Fig. 2A and an image of the device is presented in Fig. $2 \mathrm{~B}$ and $\mathrm{C}$. The device was built using different materials, including V2A steel (rods), aluminum (ground plate), teflon (upper plate), acrylic glass (ring for ground plate), rubber (rings) and brass (fixing plate and screws). Further details may be requested from the corresponding author. The device was constructed in several steps in order to avoid handling and reproducibility problems. Dummies made of plasticine (300 for development of the device and 15 used in the reported experiments) were tested to reach the required reproducibility standard. Standard drop heights were 100, 200 and $300 \mathrm{~mm}$. The effective drop heights were calculated using measurements of the top edge of the plasticine blocks or mouse cadavers, respectively. Standard formulas were used for the calculation of energy, velocity, impact and deceleration. Recoil was measured using rings of rubber on top of the rods. The rings indicate the difference between the maximum depth of 


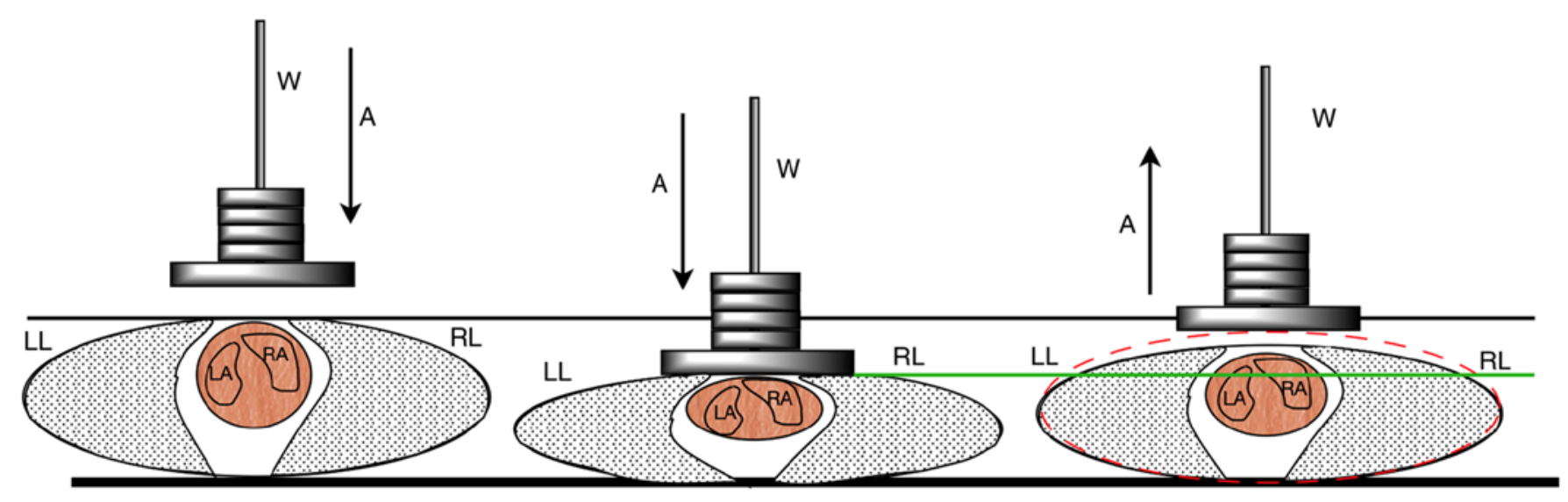

Figure 1. Sketch of the mechanism displaying the impact of $\mathrm{W}$ onto the heart with the LA, RA and both lungs. A indicates the trajectory of the weight. W, weight; LA, left atrium; RW, right atrium; LL, left lung; RL, right lung; A, arrow.

impression and the depth of impression after recoil from the impact (Fig. 2C).

Test blocks. Test blocks $(19.5 \times 19.1 \times 61.1 \mathrm{~mm})$ were made of plasticine (Pelikan Vertriebsgesellschaft mbH \& Co. KG) using a form made of acrylic glass and had a mean weight of $25 \pm 1 \mathrm{~g}$ each.

Mouse cadavers. Mouse cadavers (9; female C57BL/6 mice; weight, 23-26 g; age, 12 weeks; Charles River Laboratories, Inc.) were subjected to blunt chest trauma using the novel impact device by dropping weights from different heights within $30 \mathrm{~min}$ post mortem (at room temperature). While alive, the mice were housed in Macrolon ${ }^{\circledR}$ cages on a 12-h day/night cycle with free access to chow and water. The mice were euthanized using an overdose of pentobarbital (intraabdominal injection; $800 \mathrm{mg}$ per $\mathrm{kg}$ body weight; death confirmed by absence of breathing, heartbeat and corneal reflex) and the trauma was induced within 30-min postmortem. After trauma induction, chest X-rays were performed using a Bruker Skyscan (Bruker Corp.). Following imaging, the chests of the cadavers was carefully opened to investigate the chest organs. The cadavers were obtained following experiments that will be presented separately and were from the same control group (protocol no. 36/2015, Animal Ethical Committee of the Saarland, Homburg/Saarbrücken, Germany).

\section{Results}

Test blocks. Energy, maximum velocity (Vmax), impact, depth of impression and recoil of the plasticine blocks from the standard drop heights of 100, 200 and $300 \mathrm{~mm}$ are presented in Table I. As expected, the plasticine dummies exhibited no recoil.

Mouse cadavers. A standard falling height of $100 \mathrm{~mm}$ (effective or net falling heights of 44-46 mm, Fig. 2A), was used for the mouse cadavers to achieve impact energies from 0.13 to $0.23 \mathrm{~kg} \mathrm{~m} / \mathrm{sec}$ using falling weights of 138, 190 and $241 \mathrm{~g}$. Impact (or impulse) and other data, as well as the various depths of impression are listed in Table II.
The impact ranged from 0.13 to $0.23 \mathrm{~kg} \mathrm{~m} / \mathrm{sec}$ and depths of impression were between 12.4 and $14.7 \mathrm{~mm}$. Mouse cadaver recoils were between 0.6 and $1 \mathrm{~mm}$. In the cadaver experiments, no fractures or dislocations of the ribs were observed at any drop height, as shown in representative X-rays (Fig. 3A). By contrast, soft-tissue injuries were observed with varying characteristics: A net falling height of $45 \mathrm{~mm}$ and a drop weight of $241 \mathrm{~g}$ resulted in massive hemorrhage within the chest cavity. Furthermore, perforation/rupture of the heart right atrium was observed (Fig. 3B and C). Hemorrhage was also observed in the lung lobes. Following impact with an effective or net falling height (from lowest part of the falling weight to the highest part of the animal thorax) of $44 \mathrm{~mm}$ (gross falling height $100 \mathrm{~mm}$, for details, Fig. 2A) and a drop weight of $139 \mathrm{~g}$, the heart was normal but the lower lobe of the right lung exhibited a cleft (Fig. 3D); however, another cadaver $(44 \mathrm{~mm} / 139 \mathrm{~g})$ had no cleft or hemorrhage after the impact, but contusions/impressions from the ribs on the left and right lungs were observed (Fig. 3E and F). After an impact of medium force $(48 \mathrm{~mm} / 191 \mathrm{~g})$, one of the three mice in this experimental group exhibited a hemothorax and rupture of the right atrium, whereas the other two mice displayed no ruptures or clefts (images not shown), whereas one of them had contusions from the ribs in the right lung(images not shown but similar to those presented in Fig. 3F and E). None of the mice exhibited any visible injuries to abdominal organs.

\section{Discussion}

In the present study, a novel device for the induction of blunt chest traumata in mice was developed with the aim of improving measurement of maximal penetration depth and recoil variable energies in comparison to use of blast wave generator. In the present mouse model, no bone fractures were observed, even after large impacts. Following impact, potentially lethal injuries were observed, including rupture of the right atrium and the consecutive hemothorax. In addition, a cleft in a lung lobe would, in most cases, lead to death. To the best of our knowledge, rib-induced impressions on or contusions of the lungs may be compatible with acute survival but may also cause lung hemorrhage and lung edema, leading to death hours or days post-impact. 
A

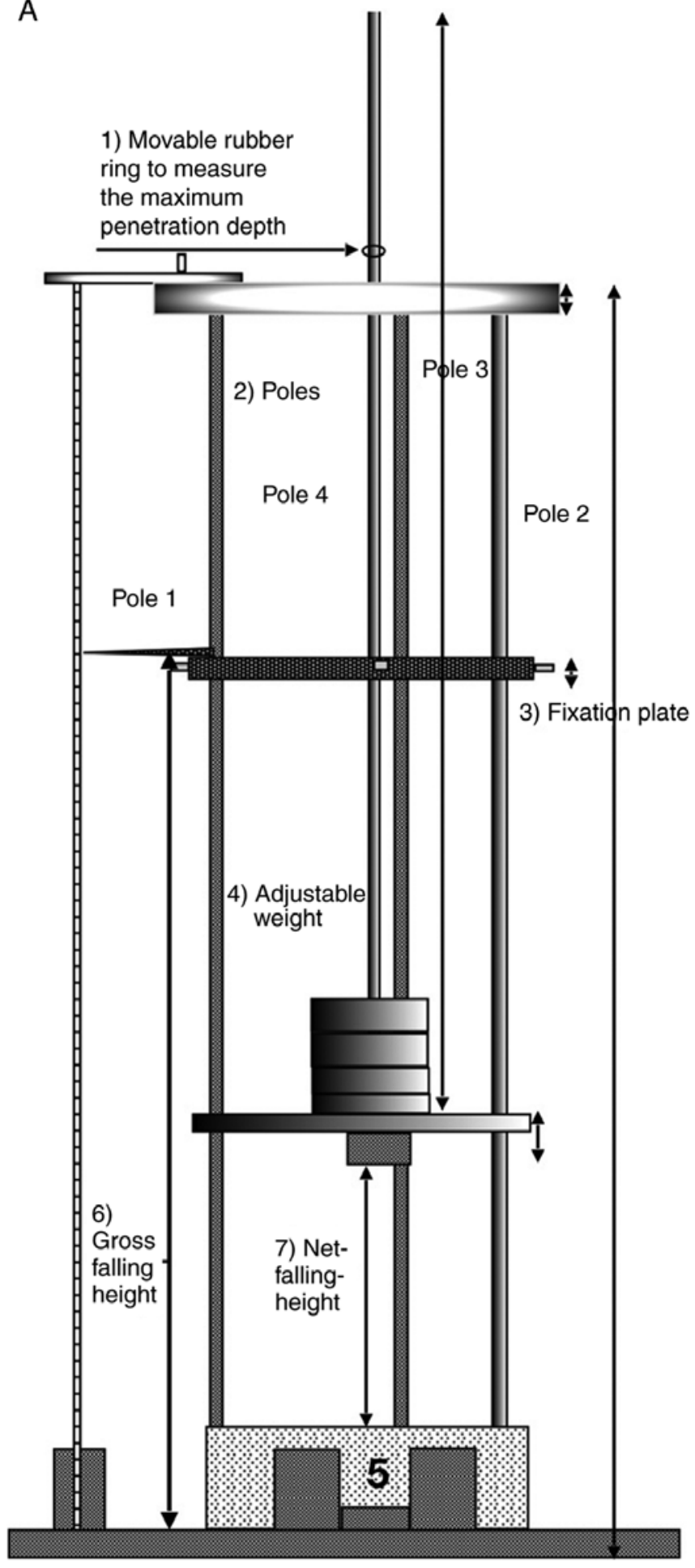

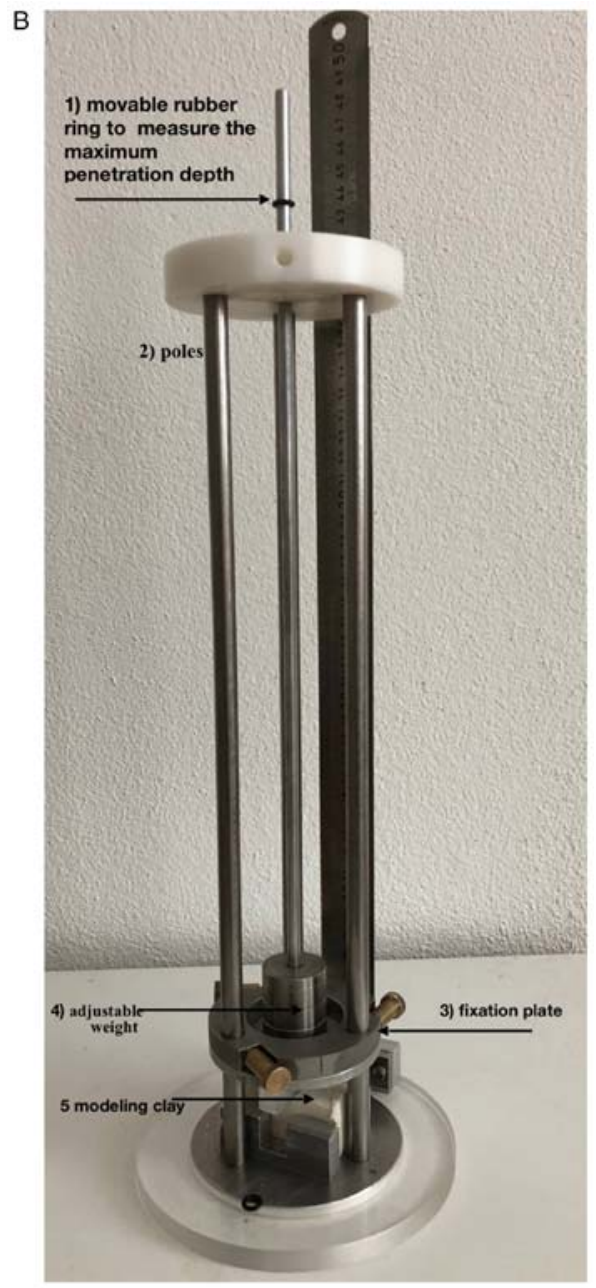

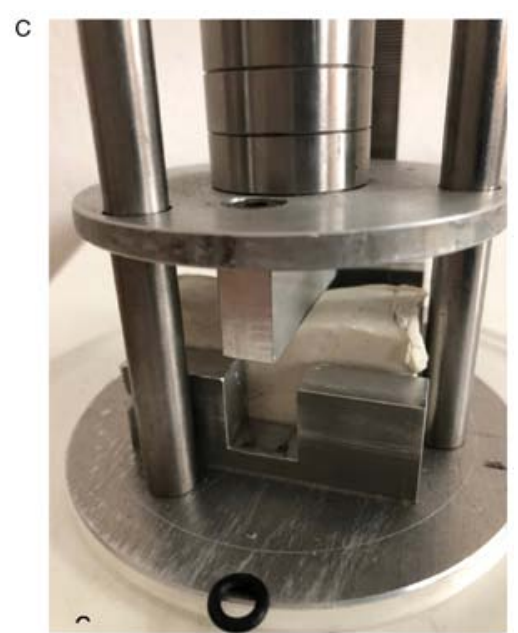

Figure 2. Novel device used for inducing blunt chest trauma. (A) Drawing depicting the design. Photographic images of (B) the device and (C) the metal weight and the plasticine block.

A noteworthy limitation of the present study is the lack of comparability between live animals and cadavers. However, the cadaver study yielded useful results. For instance, the mouse cadavers in the present study subjected to impact with an energy of $\sim 1 \mathrm{~J}$ incurred severe lesions, which would have likely been fatal in live animals. Data from a previous study using living rats demonstrated that sustaining a dropping weight impact of $2.7 \mathrm{~J}$ resulted in a mortality rate of $33 \%$ (6).
The present study concluded that an impact with an energy of $0.5 \mathrm{~J}$ would induce a severe, but probably not lethal, thorax trauma in mice. This would occur in the case of a drop height of $100 \mathrm{~mm}$ ( 44 mm effectively) and a drop weight of $\sim 110 \mathrm{~g}$. However, to the best of our knowledge, no previous study has demonstrated the lethality of an impact with an energy of $0.5 \mathrm{~J}$ in live mice. The data obtained in the present study provided a further interesting observation, namely with 


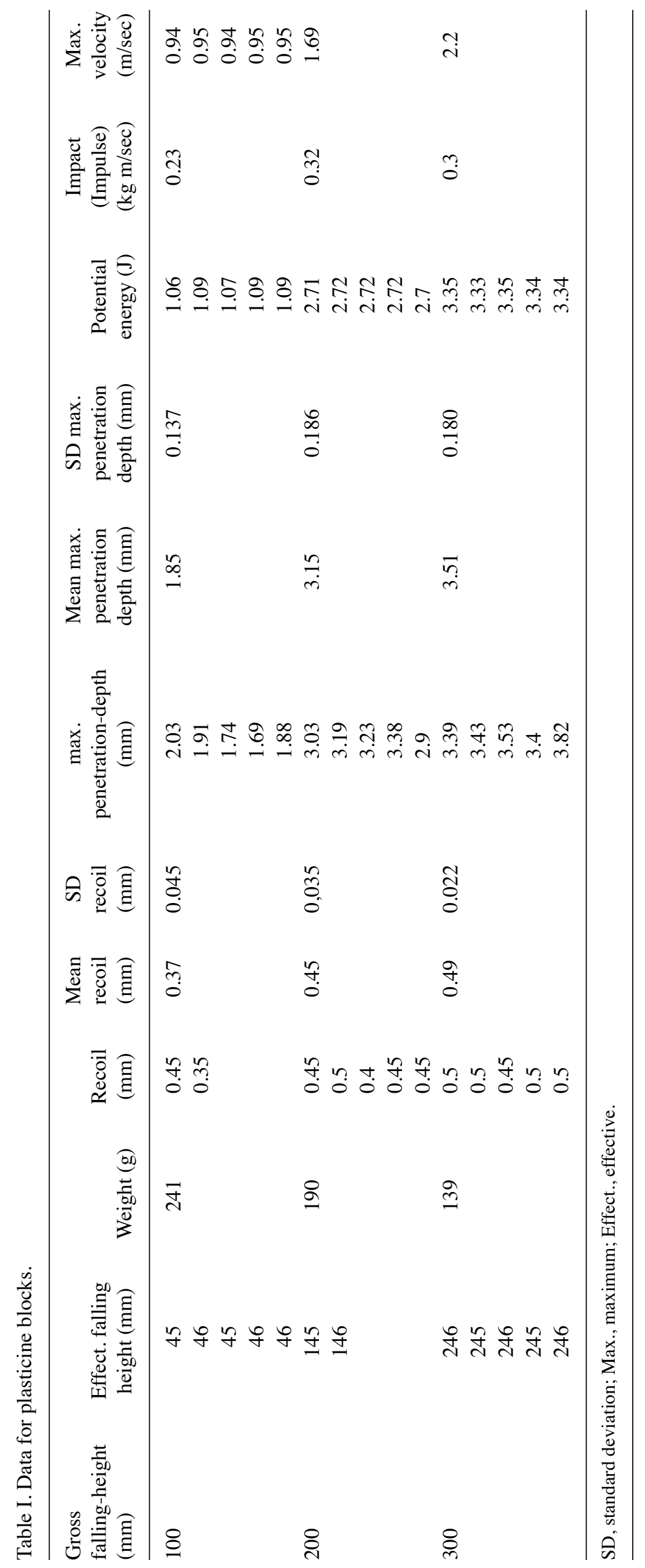


A
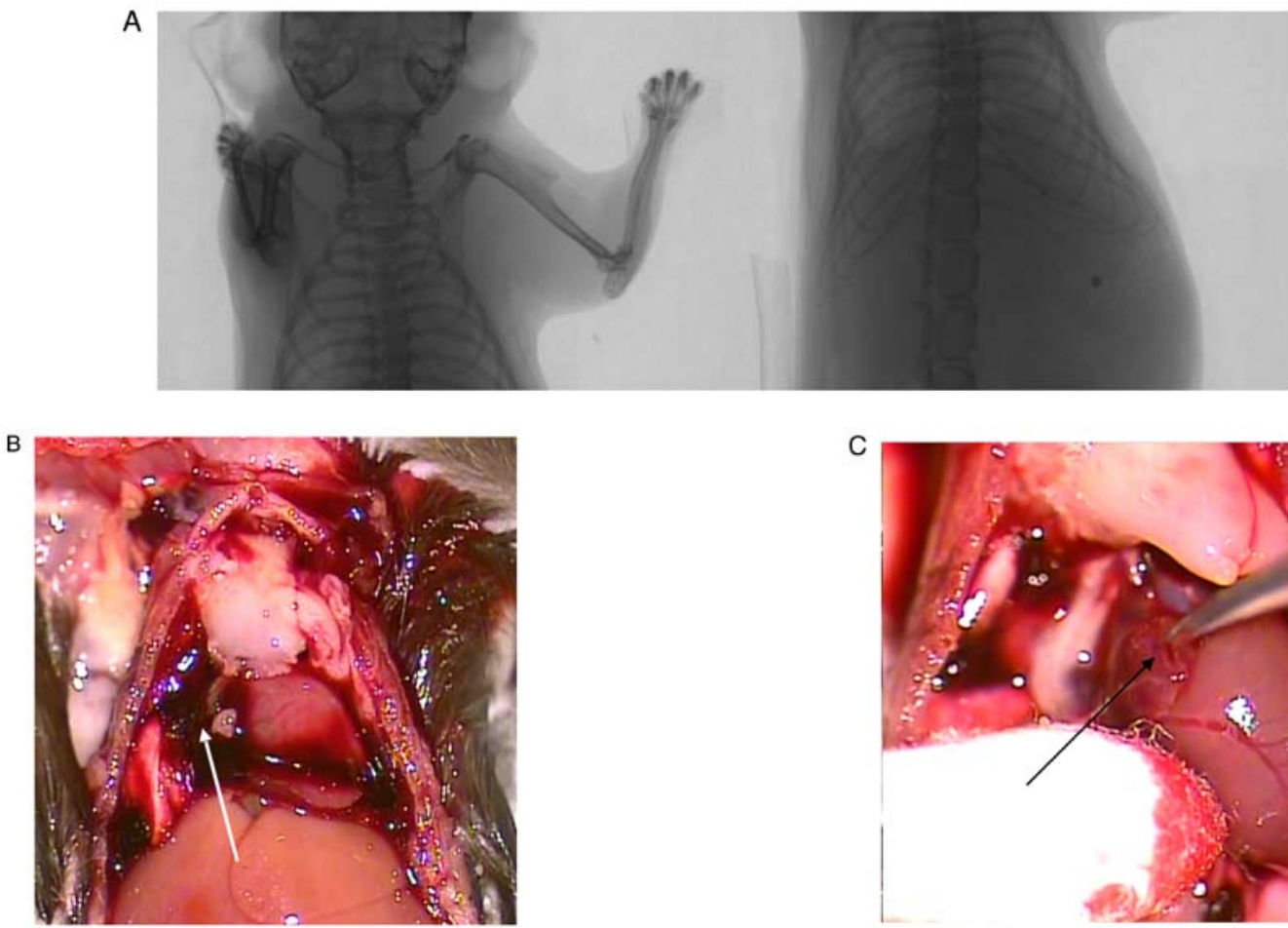

C
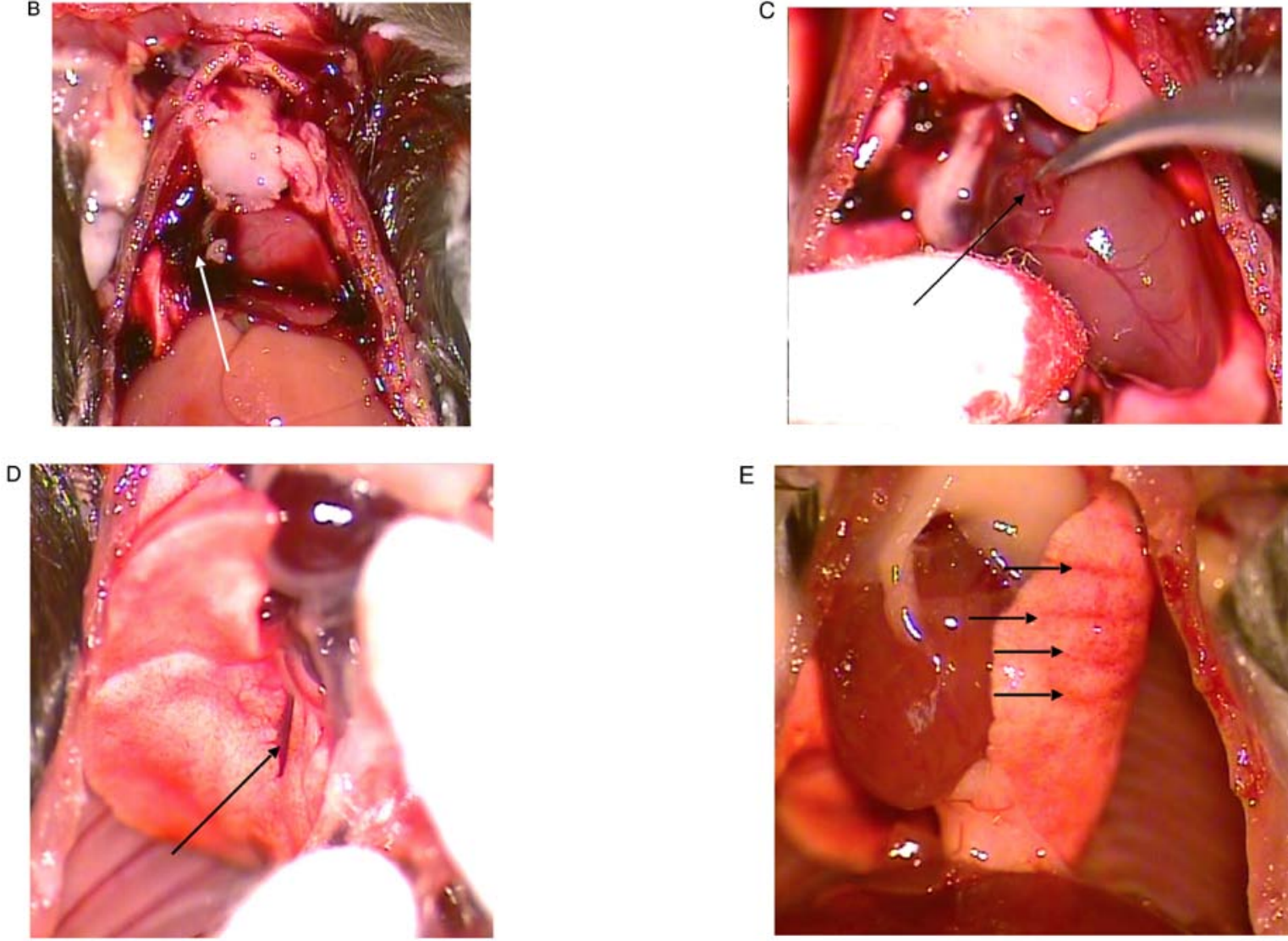

$\mathrm{E}$

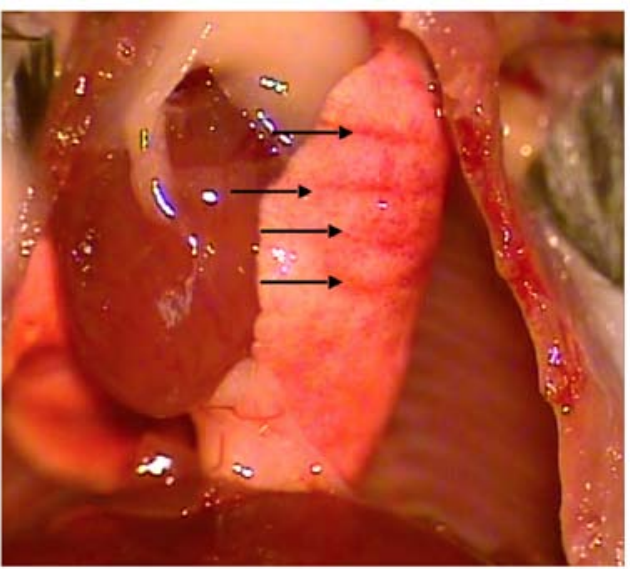

$\mathrm{F}$

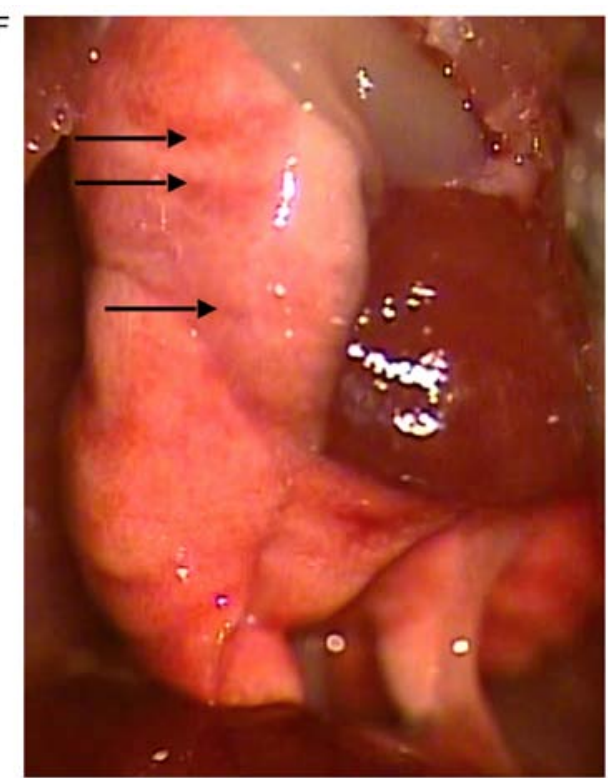

Figure 3. Findings after induction of chest trauma. (A) Chest X-ray of a mouse after trauma induction, indicating no bone lesions. Specific rather than representative images of trauma induction demonstrating (B) thoracic hemorrhage (arrow), (C) rupture of the right atrium (arrow), (D) cleft in the right lung (arrow) and impressions from ribs on (E) the left (arrows) and (F) the right lung (arrows). 


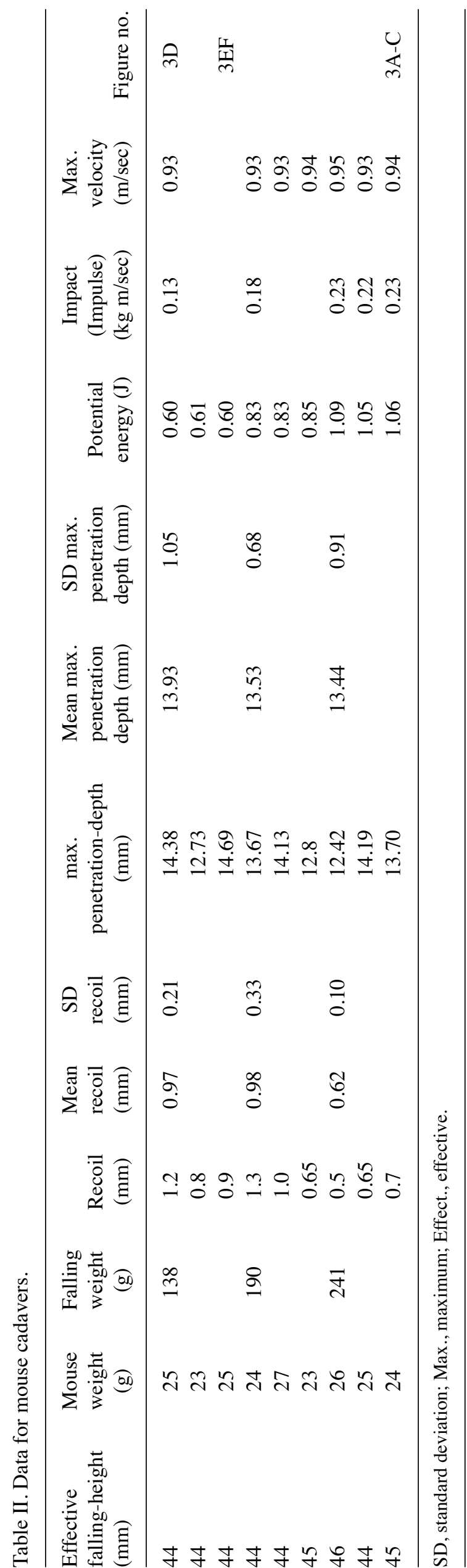

regard to the maximum penetration depth and recoil. There was no difference in the maximum penetration depth of the lightest and the heaviest drop weight in the cadaver experiments. This suggests that a threshold value or maximum of penetration depth had been reached. By contrast, the recoil tended to be reduced with heavier drop weights and would likely be running to zero with very heavy weights. The recoil forces of the animals tissues (skeleton, muscles, organs) are limited. Since a significant impact leads to a contusion of the organs underneath, the severity of the contusion of heart, lung and vessels is a complex result of the following: i) The impact energy and, ii) the maximum penetration depth. When comparing different trauma-inducing models the impact energy and the maximum penetration depth are both important in the view of the authors.

Another factor that should be taken into consideration is the shape of the impact block. If a rounded or bullet-like block is used instead of a plain block, this could influence the severity of organ lesions. The present study simulated the frontal impact such as that which would be experienced by a human struck by a car. To simulate a side impact of a vehicle on a human, the animal may be easily turned to the right side or the left side. For further translational research, it may be necessary to adapt this device for sheep, pigs or, in the case of cadaver research, even in humans. However, any device for the induction of experimental thoracic trauma and injury will only ever have a model character, as motor vehicle collisions or injuries incurred in other situations are always complex and unique. The presented model may be of use in establishing thoracic traumata for the testing of potential treatments for such trauma. Several drugs are potentially applicable for the treatment of severe but not fatal blunt chest traumata, including adrenomedullin $(7,8)$ and interleukin- 6 antagonists $(9)$, which are both suitable for the prevention of secondary lesions, such as inflammation and edema.

Overall, the device presented in the present study is a useful option complementary to the blast wave generator because maximal penetration depth and recoil can be measured and the trauma energy can be adjusted to mortality.

\section{Acknowledgements}

The authors thank the following members of the Department of Anatomy and Cell Biology (Saarland University, Campus Homburg): Mr. Jörg Sauerbaum, Mr. Christoph Neuhardt and Mr. Ronald Dollwett for building the device, Ms. Helga Meyer for providing the materials and Ms. Ann Söther for language editing.

\section{Funding}

No funding was received for the study itself, but Open Access Publishing was supported by the DFG and the Saarland University.

\section{Availability of data and materials}

The datasets used and/or analyzed during the current study are available from the corresponding author on reasonable request. 


\section{Authors' contributions}

DH, MWL, NTV, RK and TT developed the device. TT, TP and RK conducted the study. DH and CK performed the experiments. DH wrote the draft and all authors worked on the manuscript. All authors read and approved the final manuscript.

\section{Ethics approval and consent to participate}

Mouse cadavers were taken from terminated experiments (protocol no. 36/2015, Animal Ethical Committee of the Saarland, Homburg/Saarbrücken, Germany).

\section{Patient consent for publication}

Not applicable.

\section{Competing interests}

The authors declare that they have no competing interests.

\section{References}

1. Eghbalzadeh K, Sabashnikov A,Zeriouh M, Choi YH, Bunck AC, Mader $N$ and Wahlers T: Blunt chest trauma: A clinical chameleon. Heart 104: 719-724, 2018.

2. Jaffin JH, McKinney L, Kinney RC, Cunningham JA, Moritz DM, Kraimer JM, Graeber GM, Moe JB, Salander JM and Harmon JW: A laboratory model for studying blast overpressure injury. J Trauma 27: 349-356, 1987.
3. Hafner S, Wagner K, Wepler M, Matallo J, Gröger M, McCook O, Scheuerle A,Huber-Lang M,Frick M, Weber S, et al: Physiological and immune-biological characterization of a long-term murine model of blunt chest trauma. Shock 43: 140-147, 2015.

4. Fitschen-Oestern S, Lippross S, Klueter T, Weuster M, Varoga D, Tohidnezhad M, Pufe T, Rose-John S, Andruszkow H, Hildebrand F, et al: A new multiple trauma model of the mouse. BMC Musculoskelet Disord 18: 468, 2017.

5. Neunaber C, Oestern S, Andruszkow H, Zeckey C, Mommsen P, Kutter D, Stöfen M, Krettek C and Hildebrand F: Cytokine productive capacity of alveolar macrophages and Kupffer cells after femoral fracture and blunt chest trauma in a murine trauma model. Immunol Lett 152: 159-166, 2013.

6. Raghavendran K, Davidson BA, Helinski JD, Marschke CJ, Manderscheid P, Woytash JA, Notter RH and Knight PR: A rat model for isolated bilateral lung contusion from blunt chest trauma. Anesth Analg 101: 1482-1489, 2005.

7. Muller HC, Witzenrath M, Tschernig T, Gutbier B, Hippenstiel S, Santel A, Suttorp N and Rosseau S: Adrenomedullin attenuates ventilator-induced lung injury in mice. Thorax 65: 1077-1084, 2010.

8. Müller-Redetzky HC, Will D, Hellwig K, Kummer W, Tschernig T, Pfeil U, Paddenberg R, Menger MD, Kershaw O, Gruber AD, et al: Mechanical ventilation drives pneumococcal pneumonia into lung injury and sepsis in mice: Protection by adrenomedullin. Crit Care 18: R73, 2014.

9. Kang S, Tanaka $\mathrm{T}$ and Kishimoto T: Therapeutic uses of anti-interleukin-6 receptor antibody. Int Immunol 27: 21-29, 2015. 